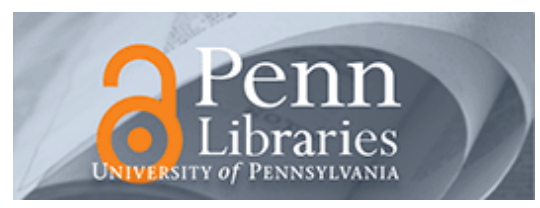

University of Pennsylvania

ScholarlyCommons

$10-15-2014$

\title{
Morphology of Rain Water Channeling in Systematically Varied Model Sandy Soils
}

Yuli Wei

University of Pennsylvania

Cesare M. Cejas

CNRS-Solvay-Penn Unité Mixte Internationale

Rémi Barrois

CNRS-Solvay-Penn Unité Mixte Internationale

Rémi Dreyfus

CNRS-Solvay-Penn Unité Mixte Internationale

Douglas J. Durian

University of Pennsylvania, djdurian@physics.upenn.edu

Follow this and additional works at: https://repository.upenn.edu/physics_papers

Part of the Physics Commons

\section{Recommended Citation}

Wei, Y., Cejas, C. M., Barrois, R., Dreyfus, R., \& Durian, D. J. (2014). Morphology of Rain Water Channeling in Systematically Varied Model Sandy Soils. Physical Review Applied, 2 (4), 044004-1-044004-12.

http://dx.doi.org/10.1103/PhysRevApplied.2.044004

This paper is posted at ScholarlyCommons. https://repository.upenn.edu/physics_papers/589

For more information, please contact repository@pobox.upenn.edu. 


\title{
Morphology of Rain Water Channeling in Systematically Varied Model Sandy Soils
}

\author{
Abstract \\ We visualize the formation of fingered flow in dry model sandy soils under different rain conditions using \\ a quasi-2D experimental setup and systematically determine the impact of the soil grain diameter and \\ surface wetting properties on the water channeling phenomenon. The model sandy soils we use are \\ random closely packed glass beads with varied diameters and surface treatments. For hydrophilic sandy \\ soils, our experiments show that rain water infiltrates a shallow top layer of soil and creates a horizontal \\ water wetting front that grows downward homogeneously until instabilities occur to form fingered flows. \\ For hydrophobic sandy soils, in contrast, we observe that rain water ponds on the top of the soil surface \\ until the hydraulic pressure is strong enough to overcome the capillary repellency of soil and create \\ narrow water channels that penetrate the soil packing. Varying the raindrop impinging speed has little \\ influence on water channel formation. However, varying the rain rate causes significant changes in the \\ water infiltration depth, water channel width, and water channel separation. At a fixed rain condition, we \\ combine the effects of the grain diameter and surface hydrophobicity into a single parameter and \\ determine its influence on the water infiltration depth, water channel width, and water channel separation. \\ We also demonstrate the efficiency of several soil water improvement methods that relate to the rain \\ water channeling phenomenon, including prewetting sandy soils at different levels before rainfall, \\ modifying soil surface flatness, and applying superabsorbent hydrogel particles as soil modifiers. \\ Disciplines \\ Physical Sciences and Mathematics | Physics
}




\title{
Morphology of Rain Water Channeling in Systematically Varied Model Sandy Soils
}

\author{
Yuli Wei, ${ }^{1,2}$ Cesare M. Cejas, ${ }^{2}$ Rémi Barrois, ${ }^{2}$ Rémi Dreyfus, ${ }^{2}$ and Douglas J. Durian ${ }^{1}$ \\ ${ }^{1}$ Department of Physics and Astronomy, University of Pennsylvania, \\ Philadelphia, Pennsylvania 19104-6396, USA \\ ${ }^{2}$ Complex Assemblies of Soft Matter, CNRS-Solvay-UPenn UMI 3254, Bristol, \\ Pennsylvania 19007-3624, USA
}

(Received 11 March 2014; revised manuscript received 13 June 2014; published 15 October 2014)

\begin{abstract}
We visualize the formation of fingered flow in dry model sandy soils under different rain conditions using a quasi-2D experimental setup and systematically determine the impact of the soil grain diameter and surface wetting properties on the water channeling phenomenon. The model sandy soils we use are random closely packed glass beads with varied diameters and surface treatments. For hydrophilic sandy soils, our experiments show that rain water infiltrates a shallow top layer of soil and creates a horizontal water wetting front that grows downward homogeneously until instabilities occur to form fingered flows. For hydrophobic sandy soils, in contrast, we observe that rain water ponds on the top of the soil surface until the hydraulic pressure is strong enough to overcome the capillary repellency of soil and create narrow water channels that penetrate the soil packing. Varying the raindrop impinging speed has little influence on water channel formation. However, varying the rain rate causes significant changes in the water infiltration depth, water channel width, and water channel separation. At a fixed rain condition, we combine the effects of the grain diameter and surface hydrophobicity into a single parameter and determine its influence on the water infiltration depth, water channel width, and water channel separation. We also demonstrate the efficiency of several soil water improvement methods that relate to the rain water channeling phenomenon, including prewetting sandy soils at different levels before rainfall, modifying soil surface flatness, and applying superabsorbent hydrogel particles as soil modifiers.
\end{abstract}

DOI: 10.1103/PhysRevApplied.2.044004

Improving the usage of rain and irrigation water by plants in sandy soils is an important topic in agriculture, which draws increasing attention with the reduction of the water supply and growth of the human population. Sandy soils store water mainly through a capillary effect-their pores capture and lock a small amount of water by capillary forces when rain or irrigation water flows through them. Previous studies [1-4] show that superabsorbent hydrogel particle additives can significantly decrease the water conductivity and enhance water retention in sandy soils. However, these studies were conducted in ideal fully saturated soil systems, which significantly differ from a real situation in plant root zones commonly containing partially wet or dry soils. Early laboratory experiments [5-8] on water infiltration studies observed the formation of fingered flows in dry layered sands under uniform water flow onto the top sand layer. Later, field studies [9-13] demonstrated the existence of preferential water paths in sandy soils during rainfall or irrigation. At the same time, laboratory experiments [14-18] further confirmed that rain water channeling is a common feature that widely exists not only in sandy soils with structure heterogeneity, but also in uniform dry sands with almost no structure defects. The cause of the latter is due to instabilities that occur at the gravity-driven water wetting front $[14,16]$. Rainwater channeling largely reduces the water-reachable area in the plant root zone and results in a significant deviation of the predicted soil water capacity from measurements, which are usually started or performed at a fully saturated state. Therefore, developing new techniques to incorporate the water channeling phenomenon into the evaluation of soil water capacity and soil additive efficiency is crucial to achieve more reliable and applicable results. To do so, there is an increasing need for characterizing the morphology of channeling and understanding how it is affected by rain and soil properties.

This paper focuses on the morphology of rain water channeling. After describing the experimental setup, we examine rain water channeling using systematically varied dry model sandy soils with well-controlled grain diameters and surface wetting properties. A quasi-2D setup is built to mimic a steady rainfall and to capture the formation of water channels. In a steady state, the key parameters (including the water infiltration depth, water channel width, and water channel separation) are determined for each soil sample and then plotted against the rain conditions (raindrop impinging speed and rain rate) and soil properties (grain diameter and surface hydrophobicity). Lastly, we discuss irrigation efficiency improvement methods that relate to the rain water channeling phenomenon and demonstrate their effectiveness under different circumstances. In a companion paper, we study the kinetics of rain water channeling [19]. 


\section{EXPERIMENT}

For reproducible model sandy soils, we use monodisperse solid glass beads with diameters varying from $D=0.18 \mathrm{~mm}$ to $1 \mathrm{~mm}$ (A-series, Potters Industries Inc.). To clean the glass beads, they are first burned in a furnace at $500{ }^{\circ} \mathrm{C}$ for $72 \mathrm{~h}$ and then soaked in a $1 \mathrm{M} \mathrm{HCl}$ bath for an hour. After that, the beads are rinsed with deionized water, baked in a vacuum oven at about $110^{\circ} \mathrm{C}$ for $12 \mathrm{~h}$, and then cooled to room temperature in air. The clean samples have hydrophilic surfaces - our tests show that the contact angle of water on a clean glass bead surface is $\theta^{*}=$ $16^{\circ} \pm 2^{\circ}$. Through additional chemical treatments described below, we modify the surface wetting property of the clean glass beads to be hydrophobic. Our tests confirm that the contact angle of water on a treated glass bead surface is around $90^{\circ}$. Mixing treated beads into the clean ones changes the effective contact angle of the whole packing, and the way we determine the effective contact angle of a soil packing [see Eq. (3)] is to use a set of independent capillary rise experiments rather than the contact angle measurement on a single glass bead.

We make two different size sample cells to hold the glass beads. A 26-cm-wide and 30-cm-high sample cell is used when probing the effect of the soil grain diameter; a $55-\mathrm{cm}-$ wide and $15-\mathrm{cm}$-high sample cell is chosen when probing the effect of soil surface wetting properties. All the sample cells are made of two parallel sheets of hydrophobic glass with a separation of $e=0.8 \mathrm{~cm}$. The bottom of each sample cell is covered by several layers of mesh which hold soil grains inside the sample cell well but allow air to freely circulate in and out of the soil packing during rain. Rain water can also freely drain out through the meshes. The sample cell is cleaned and dried before each experiment. Glass bead samples are then poured carefully into the cell. During the pouring, we pat the cell gently from time to time to ensure a random close packing. The volume fraction of the glass beads is measured to be between 0.60 and 0.64 .

Figure 1 shows a schematic of the front view of our experimental setup. A sample cell is suspended under a 2D rain source built by inserting a line of glass capillaries ( 5 to $50 \mu \mathrm{l}$ borosilicate micropipet, Kimble Inc.) in the bottom of a plastic container with a separation of $1 \mathrm{~cm}$. Rain rate $Q$ in units of $\mathrm{cm} / \mathrm{h}$ is defined as the volume of rain water per unit time per unit cross-sectional area of the sample. The value is determined by measuring the mass of falling rain water within one minute several times. It shows a linear relationship with the water level in the plastic container. A gear pump (Micropump Inc.) is used to maintain a constant water level in the plastic container. The diameter of the rain droplets is estimated by their average mass to be around $3 \mathrm{~mm}$. The impinging speed $U_{T}$ of rain droplets depends on the free-falling distance $h$ of rain droplets and is estimated as

$$
U_{T}=\sqrt{2 g h}
$$

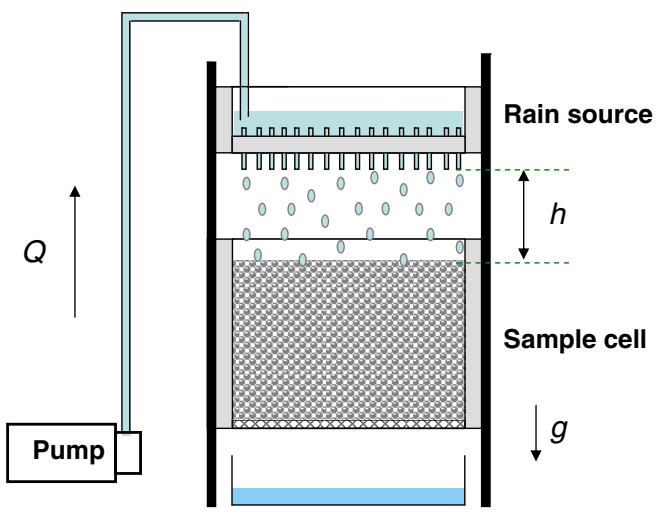

FIG. 1. Schematic of the experimental setup for visualizing rain water channeling in model sandy soils. A sample cell partially filled with model sandy soils is suspended under a $2 \mathrm{D}$ rain source built by a linear array of glass capillaries inserted into the bottom of a plastic container with a separation of $1 \mathrm{~cm}$. The sample cell can be shifted up or down to adjust the falling distance $h$ of the rain droplets. Rain rate $Q$ is determined by the water level in the plastic container. A gear pump is used for supplying water during the experiment.

where $g$ is the gravitational acceleration. The location of the sample cell can be shifted up or down to adjust the value of $h$ and, thus, to vary the impinging speed $U_{T}$ of the rain droplets.

During the experiments, we illuminate the sample cell from the back using a light box of the same size as the sample cell, and we take images from the front using a digital camera (Nikon D90). The camera is controlled by a computer through a LABVIEW program, which allows us to automatically record an image sequence at a preset frame rate.

\section{WATER CHANNELING IN MODEL SANDY SOILS}

We begin with hydrophilic samples with varying grain diameters at different rain conditions. Similar observations are obtained for these tests as described below and as demonstrated in Fig. 2. Rain water infiltrates a shallow top layer of soils to create a fully saturated region with a horizontal wetting front. As time goes on, the wetting front moves downward and the hydraulic pressure across it keeps increasing. When it exceeds the capillary forces in the model sandy soils, instabilities occur on the wetting front and grow to form water channels that penetrate through whole soil packing. After that, rain water keeps flowing out of the soil packing through water channels, and the system reaches a steady state. See Ref. [19] for a detailed characterization of this time-evolution kinetics; here we focus on the steady state. We also notice that during and after the formation of water channels, the wet regions (including the wet top layer and water channels) may become partially saturated. The location of the water channels at different test runs varies, but 
(a) $D=0.18 \mathrm{~mm}$

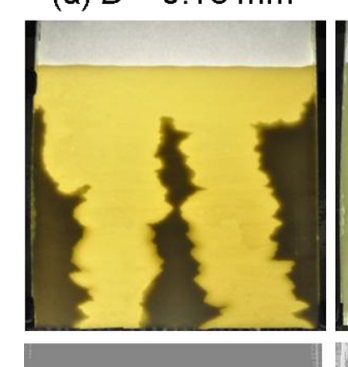

(b) $D=0.3 \mathrm{~mm}$
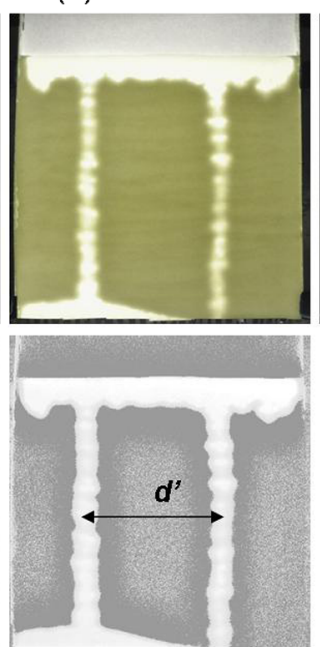

(c) $D=0.5 \mathrm{~mm}$

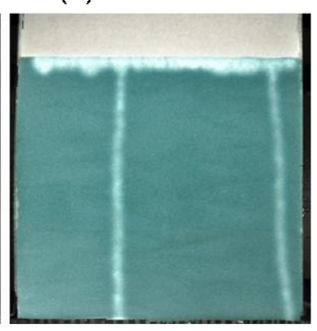

(d) $D=1.0 \mathrm{~mm}$

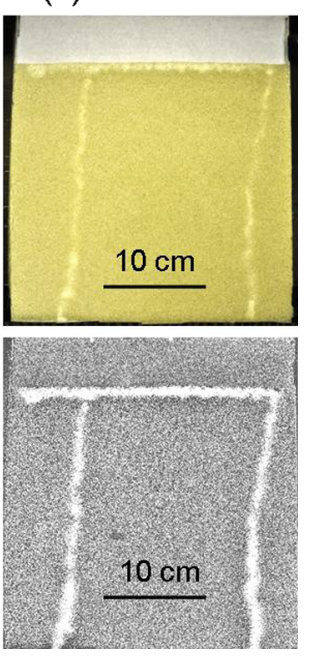

FIG. 2. Images (a)-(d) show deionized-water channeling in dry hydrophilic model sandy soils with bead diameters $D$ varying from 0.18 to $1 \mathrm{~mm}$, at a rain rate of $Q=14.5 \mathrm{~cm} / \mathrm{h}$ and a raindrop impinging speed of $U_{T}=1 \mathrm{~m} / \mathrm{s}$. The color images in the first row are the original images taken at steady states; the gray-scale images in the second row are obtained by subtracting the background images taken prior to the rain from the ones taken at steady states, converting to gray scale, and then enhancing the contrast. The sample packing in each image is $26 \mathrm{~cm}$ wide, $25 \mathrm{~cm}$ high, and $0.8 \mathrm{~cm}$ thick. As labeled in the images, $z_{\text {wet }}$ is the infiltration depth of rain water in soils, $d$ is the water channel width, and $d^{\prime}$ is the water channel separation. The saturated hydraulic conductivity $\kappa_{s}$ [Eq. (2)] for these four samples are determined as $73 \mathrm{~cm} / \mathrm{h}, 204 \mathrm{~cm} / \mathrm{h}, 567 \mathrm{~cm} / \mathrm{h}$, and $2300 \mathrm{~cm} / \mathrm{h}$, respectively.

the separation between channels is very similar. The use of different-width sample cells excludes the effects of sample cell size. As an example, in a 26-cm-wide packing of 1-mm hydrophilic glass beads, we see either one water channel form near the center or two water channels form close to each side; in a 55-cm-wide packing of 1-mm hydrophilic glass beads, we see four to six water channels form with similar separation.

Figures 2(a)-2(d) show typical steady states of rain water channeling in dry hydrophilic model sandy soils with varying bead diameters. The applied rainfall rate is $Q=14.5 \mathrm{~cm} / \mathrm{h}$, far smaller than the saturated hydraulic conductivity of soils. The top row consists of raw images taken after a steady state is achieved. The bottom row shows gray-scale images obtained by subtracting the background images taken before the rain started from the raw ones in the top row, converting the result to gray scale, and then enhancing the contrast using a histogram equalization algorithm provided by MATLAB. The three parameters that we are interested in are labeled on the processed gray-scale images of the figure. They are the infiltration depth $z_{\text {wet }}$ of the wetting front, the channel width $d$, and the channel separation $d^{\prime}$. In the following subsections, we discuss the effects of rain conditions and sandy soil properties on these parameters, respectively.

\section{A. Effects of raindrop impinging speed and rain rate}

We first study the influence of the rain conditions, such as the raindrop impinging speed $U_{T}$ and rain rate $Q$, using 1-mm dry hydrophilic glass bead packing. Both deionized-water and glycerol-water mixtures are used as the rain water supply so that the effects of rain water quality can also be investigated. An important parameter called the saturated hydraulic conductivity is widely used in these studies. It quantitatively shows how fast a fluid can move through the pore spaces in a soil. For the model sandy soils we use, the value of the saturated hydraulic conductivity $\kappa_{s}$ can be determined as

$$
\kappa_{s}=\frac{\rho g}{\mu} K_{0} D^{2},
$$

where $\rho$ and $\mu$ are the density and the viscosity of the applied fluid, respectively, $D$ is the soil grain diameter, and $K_{0}$ is the intrinsic permeability of the soil. For a random close packing of spheres with a porosity of $\epsilon \approx 0.36, K_{0}$ has a value of $6.3 \times 10^{-4}[3,20]$. In theories of channeling, the rain rate often enters as the dimensionless ratio $Q / \kappa_{s}$, and experiments are performed at variable $Q$. Here, we vary $Q$, but we also shall vary the fluid viscosity as an alternative means of achieving a change in $Q / \kappa_{s}$.

When impinging on sandy soils, rain droplets create craters on the soil surface, whose size and shape depend strongly on both the impinging speed and the soil grain diameter [21,22]. To determine if the craters affect the stability of the horizontal wetting front and, thus, control the locations of the water channels, we vary the raindrop impinging speed $U_{T}$ by adjusting the distance $h$ between the capillary tips on the rain source and the soil surface at two fixed rain rates, $Q=14.5 \mathrm{~cm} / \mathrm{h}$ and $Q=96.0 \mathrm{~cm} / \mathrm{h}$. When a low rain rate of $Q=14.5 \mathrm{~cm} / \mathrm{h}$ is applied, 
deionized water is used as "rain water," and the saturated hydraulic conductivity in a model sandy soil is determined to be $\kappa_{s}=2300 \mathrm{~cm} / \mathrm{h}$ using Eq. (2). The ratio of the rain rate over the soil saturated hydraulic conductivity is only around 0.006 for these cases. Similar experimental observations are obtained for all $U_{T}$ values that we test, as described before. Quantitatively, as shown in Figs. 3(a)-3(c), there is no obvious difference in the infiltration depth $z_{\text {wet }}$, channel width $d$, and channel separation $d^{\prime}$, as the value of $U_{T}$ increases at a fixed low rain rate of $Q=14.5 \mathrm{~cm} / \mathrm{h}$. The reason is that the infiltration depth in model sandy soils is larger than the size of the craters. When a high rain rate of $Q=96.0 \mathrm{~cm} / \mathrm{h}$ is applied, a $40 \%$ glycerol-water mixture [23] is used as rain water, and the saturated hydraulic conductivity in the same model sandy soil is reduced to $\kappa_{s}=620 \mathrm{~cm} / \mathrm{h}$ due to the higher

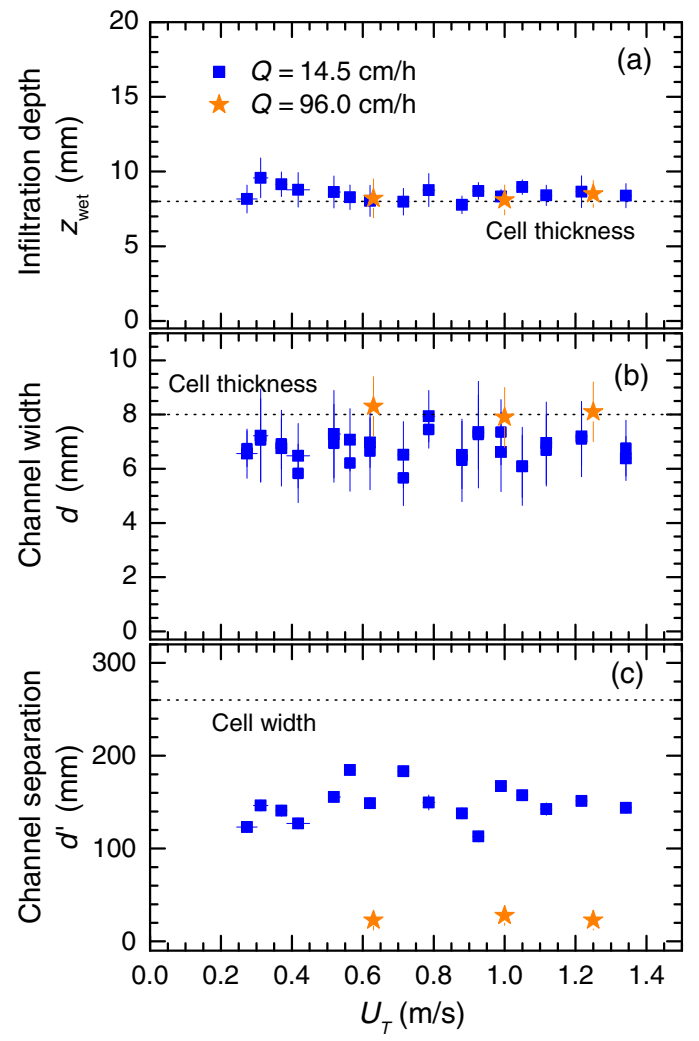

FIG. 3. Variation of (a) infiltration depth $z_{\text {wet }}$, (b) channel width $d$, and (c) channel separation $d^{\prime}$, with the raindrop impinging speed $\left(U_{T}\right)$ in a dry model sandy soil of $D=1 \mathrm{~mm}$ hydrophilic glass beads at two fixed rain rates. The parameters are defined in Fig. 2. The raindrop impinging speed is estimated from the falling height of the rain droplets using Eq. (1). When a low rain rate of $Q=14.5 \mathrm{~cm} / \mathrm{h}$ is applied, deionized water is used as rain water, and the saturated hydraulic conductivity in the model sandy soil is determined to be $\kappa_{s}=2300 \mathrm{~cm} / \mathrm{h}$ [Eq. (2)]. When a high rain rate of $Q=96.0 \mathrm{~cm} / \mathrm{h}$ is applied, a glycerol-water mixture is used as rain water, and the corresponding saturated hydraulic conductivity in the same model sandy soil is reduced to $\kappa_{s}=$ $620 \mathrm{~cm} / \mathrm{h}$ [Eq. (2)]. viscosity of the rain water. The ratio of the rain rate over the soil saturated hydraulic conductivity now rises to around 0.16 , but the experimental observation for different $U_{T}$ values is still roughly the same. Figures 3(a)-3(c) again show that the raindrop impinging speed has no influence on infiltration depth $z_{\text {wet }}$, channel width $d$, and channel separation $d^{\prime}$ at a high rain rate. The reason is the same as that given for the low-rain-rate cases.

Note that the cell thickness and the cell width are also marked by dotted lines in the figures so that the dimension of the water channels can be easily compared. In Fig. 3(a), most of the data points just coincidentally overlap with the dotted line of the cell thickness. In Fig. 3(b), the data points are equal to or slightly lower than the dotted line of the cell thickness, which tells us that the water channels should be considered as three dimensional. In Fig. 3(c), all data points are lower than the cell width, which indicates that the cells are wide enough to allow multiple water channels to form simultaneously.

Unlike the raindrop impinging speed that has no influence at all, the rain rate tells a very different story. We notice that in Fig. 3(b) the channel width is slightly larger when a higher rain rate is applied, and in Fig. 3(c), the channel separation is dramatically reduced at a higher rain rate. To further clarify the influence of the rain rate, we fix the raindrop impinging speed to $U_{T}=1.0 \mathrm{~m} / \mathrm{s}$ and vary the rain rate $Q$ by changing the water level in the water container. Yao and Hendrickx [16] reported unusual water channel size changes at extremely low rain rates using real sands with different grain size ranges. Here, we focus on a relatively high range of rain rates, from $12 \mathrm{~cm} / \mathrm{h}$ to $220 \mathrm{~cm} / \mathrm{h}$, and apply different types of rain water in a model sandy soil with well-known pore structure. Since many theory studies [24-28] suggested that the relation between the rain rate and the soil hydraulic conductivity played an important role in the formation and the size of the water channels, we scale the applied rain rate $Q$ with the saturated hydraulic conductivity of soil $\kappa_{s}$ in our plots to see if we can collapse the data obtained from different types of rain water. As shown in Fig. 4, in addition to deionized water, a $40 \%$ glycerol-water mixture and a $50 \%$ glycerolwater mixture are applied in the experiments. Their viscosity is about 4 times and 8 times higher than deionized water, respectively, but their density and surface tension are still very close to deionized water (changes usually within 10\%) [23,29]. Based on Eq. (2), using glycerol-water mixtures to replace deionized water in the experiments reduces the hydraulic conductivity in the same model sandy soil to be $1 / 4$ and $1 / 8$, respectively, and easily extend the range of $Q / \kappa_{s}$ to be close to 1 .

We observe water channel formation in all the experiments with varying channel position, size, and number. In Fig. 4(a), we see that the infiltration depths obtained from different testing liquids collapse in a certain region. As a whole, they show a strong dependence on the value of $Q / \kappa_{s}$ 


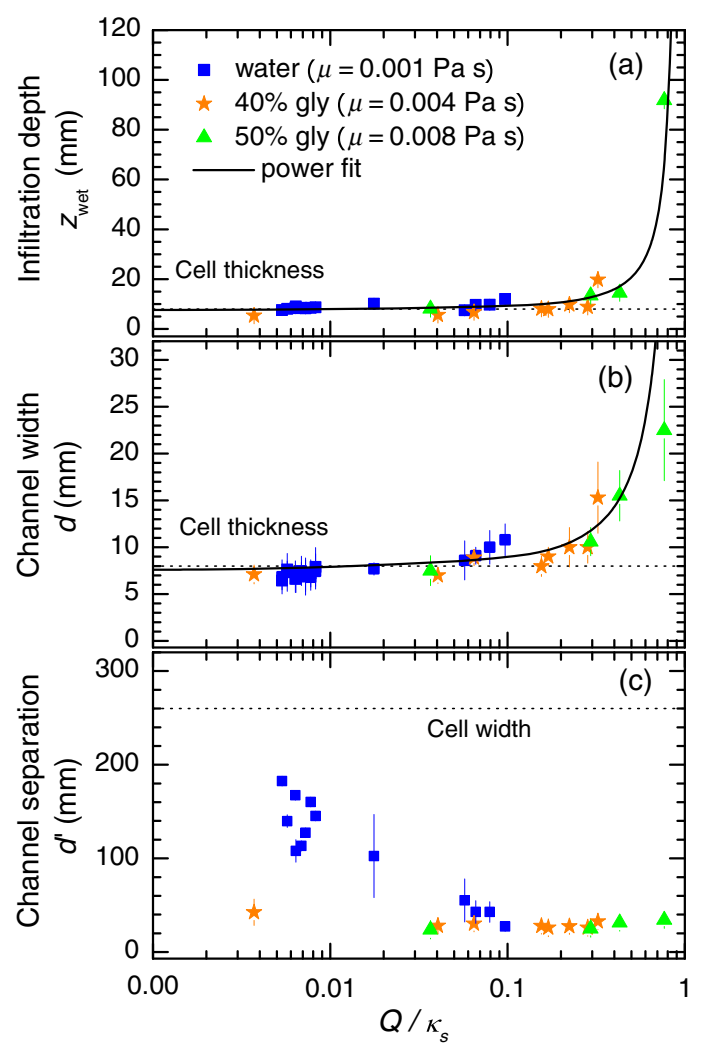

FIG. 4. Variation of (a) infiltration depth $z_{\text {wet }}$, (b) channel width $d$, and (c) channel separation $d^{\prime}$, with the rain rate $(Q)$ and rain water viscosity $\mu$ in a dry model sandy soil of $D=1 \mathrm{~mm}$ hydrophilic glass beads, at a fixed raindrop impinging speed $U_{T}=1.0 \mathrm{~m} / \mathrm{s}$. The parameters are defined in Fig. 2 . The rain rate $Q$ is scaled by the saturated hydraulic conductivity $\kappa_{s}$ of the model sandy soil. Three different liquids are used as rain water to extend the testing range: deionized water, a $40 \%$ glycerol-water mixture, and a $50 \%$ glycerol-water mixture. Their corresponding saturated hydraulic conductivities in the same model sandy soil are determined to be $\kappa_{s}=2300 \mathrm{~cm} / \mathrm{h}, \kappa_{s}=620 \mathrm{~cm} / \mathrm{h}$, and $\kappa_{s}=326 \mathrm{~cm} / \mathrm{h}$, respectively [Eq. (2)]. Solid lines in (a) and (b) are power-law fits to the data.

and rise dramatically as the value of $Q / \kappa_{s}$ approaches 1 . It is reasonable since theoretically the stable horizontal wetting front should smoothly move downward forever when the supply water matches the saturated hydraulic conductively in a soil. When the supply water is less than the soil saturated hydraulic conductivity, there is no need for all the soil to get wet to conduct the supply water; thus, instabilities may occur on the wetting front and grow to be water channels. We fit the data in Fig. 4(a) to a power law of $a\left(1-Q / \kappa_{s}\right)^{-\Delta}$ with $a$ and $\Delta$ as the fitting parameters, determining that $\Delta=1.4 \pm 0.5$, as shown by the solid line in the figure. The uncertainty reflects the scatter in exponents when the four largest $Q / \kappa_{s}$ data points are progressively excluded from the fit.

The effects of varying $Q / \kappa_{s}$ on the water channel width is even more obvious. In Fig. 4(b), the channel width data obtained from different viscosity liquids again collapse together and also show a power-law increase as the $Q / \kappa_{s}$ value increases. We fit the data in Fig. 4(b) to the same power-law equation of $b\left(1-Q / \kappa_{s}\right)^{-\Delta^{*}}$ with $b$ and $\Delta^{*}$ as the fitting parameters and obtain a value of $\Delta^{*}=1.2 \pm 0.4$. Again, the uncertainty reflects the scatter in exponents when the four largest $Q / \kappa_{s}$ data points are progressively excluded from the fit. Also, compared to the dotted line of the cell thickness, most of the water channels we obtain are 2D.

Previous theoretical studies by Chuoke et al. [24] showed that the water channel width followed this type of power law with a value of $\Delta^{*}$ equal to $1 / 2$. Using a different analysis method, Parlange and Hill [25] later predicted that the water channel width followed the same power law, but the value of $\Delta^{*}$ equals 1 . Since both Chuoke's model and Parlange's model aimed at real sandy soils, they used a measured hydraulic conductivity $\kappa_{f}$ of water channels rather than the saturated hydraulic conductivity $\kappa_{s}$ in their expressions [see Eqs. (4) and (5)]. For the model sandy soils we deal with, $\kappa_{f} \approx \kappa_{s}$ is a good assumption. Comparing the values of $\Delta^{*}$, we find that our result lies coincidentally between Chuoke's model and Parlange's model. It is hard to say whose prediction may be better from here. More detailed discussion regarding their models is given later in this paper when examining the effects of the soil grain diameter and surface wetting property and also in our kinetic study paper [19].

In Fig. 4(c), an interesting thing we notice is that the channel separation data obtained from deionized water at low $Q / \kappa_{s}$ values no longer collapse with those obtained from glycerol-water mixtures at similar $Q / \kappa_{s}$ values. We believe the slight reduction of the surface tension in the glycerol-water mixtures compared to deionized water may contribute to this phenomenon. When deionized water is applied in the experiments, we see that the number of channels formed in the sample cell increases quickly from two to nine as rain rate $Q$ increases from $12 \mathrm{~cm} / \mathrm{h}$ to $220 \mathrm{~cm} / \mathrm{h}$. At the same time, the changes on the channel width are relatively small, from only about 7 to $11 \mathrm{~mm}$. The significant change on the water channel number largely reduces the separation between the channels, and, thus, we see a decrease in the water channel separation in Fig. 4(c). When glycerol-water mixtures are applied in the experiments, the number of channels formed in the sample cell varies between six and ten as $Q$ increases from $2.3 \mathrm{~cm} / \mathrm{h}$ to $250 \mathrm{~cm} / \mathrm{h}$. However, in the same $Q$ range their channel width is double or even triple. Therefore, in Fig. 4(c), the channel separation obtained from glycerol-water mixtures is roughly the same at different $Q / \kappa_{s}$ values.

\section{B. Effects of grain diameter and surface wetting properties}

In this section the rain conditions are fixed to $Q=$ $14.5 \mathrm{~cm} / \mathrm{h}$ and $U_{T}=1.0 \mathrm{~m} / \mathrm{s}$ so that we can focus on the effects of varying the soil grain diameter or surface wetting 
properties. Since the pore size of the sandy soils is proportional to their grain diameter, increasing the grain diameter $D$ enlarges the soil pores and lowers the capillary forces in sandy soils. In Figs. 2(a)-2(d), we see that varying the grain diameter $D$ significantly changes the infiltration depth of the rain water and the width of the water channel as well. However, the number of water channels formed in the same size sample cell remains the same, and the channel separation is similar. The quantitative results are plotted later in Fig. 6 using solid squares.

In addition to the soil pore size, the surface wetting property also controls the capillary forces in sandy soils. When the soil grains become partially hydrophobic, the capillary forces in the soil pores drop quickly. To determine the effects of soil hydrophobicity, we prepare partially hydrophobic samples by treating a small amount of clean 1-mm glass beads with a hydrophobic polymer solution (OMS Opto Chemicals, Montreal, Canada) and then uniformly mixing them into the same diameter untreated clean beads at different percentages. Our tests show that the contact angle of water on a single treated bead is around $90^{\circ}$. The hydrophobicity of the mixtures is determined by separate capillary rise experiments, similar to those employed in Ref. [30]. In capillary rise experiments, the airdried mixtures are poured into hydrophobic glass tubes with meshes covering the bottoms. Then the tubes are vertically placed in a shallow water reservoir for about two days. The difference of the water levels in the glass bead packing and in the reservoir gives us the value of the capillary rise height $H$. We measure that the contact angle of the hydrophilic beads is $\theta_{0}^{*}=16^{\circ} \pm 2^{\circ}$. We also use its capillary rise height value $H_{0}$ as the reference to determine the effective contact angle for the mixture packing with both the clean and treated beads:

$$
\cos \left(\theta^{*}\right)=\cos \left(\theta_{0}^{*}\right) \frac{H}{H_{0}}
$$

From Eq. (3), a glass bead packing of completely hydrophilic beads will have $\cos \left(\theta^{*}\right) \neq 1$ since the glass beads themselves are not perfectly hydrophilic, as revealed from the measured contact angle value. Furthermore, the capillary rise results for the mixtures are shown in Fig. 5. From the figure, we see that the value of $\cos \left(\theta^{*}\right)$ decreases linearly as the percentage of treated beads increases. When the treated beads in the mixture reach around 30\%, the capillary rise height $H$ in the mixture decreases to zero, and the mixture has an effective contact angle of $\theta^{*}=90^{\circ}$.

Under rainfall, the behavior of the partially hydrophobic samples differs from that of very hydrophilic ones. When the percentage of treated beads in a mixture is no more than $15 \%\left[\cos \left(\theta^{*}\right)=0.44\right]$, we still see that rain water infiltrates a top layer of soil samples and creates a horizonal wetting front. After that, water channels form due to the instabilities, and the system reaches a steady state. However, the

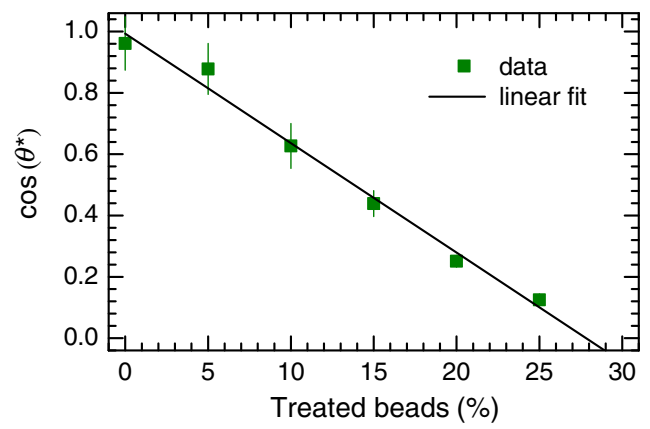

FIG. 5. Variation of the effective contact angle $\left[\cos \left(\theta^{*}\right)\right]$ with the percentage of hydrophobic beads. The linear fit goes from complete wetting for untreated beads, to zero for a mixture of $30 \%$ treated beads.

infiltration depth in these cases is far shallower, and the wetting front is not as flat and smooth as that seen in the very hydrophilic case. Also, a small change on soil hydrophobicity significantly increases the number of water channels that form in dry soils under rainfall. For example, in a 55-cm-wide packing of glass beads, when treated beads change from 0 to $15 \%$, the number of water channels increases from four to ten. For these cases, we try our best to extract their infiltration depth, channel width, and channel separation. The obtained values are also added to Fig. 6 in open stars.

When treated beads in a mixture exceed $20 \%\left[\cos \left(\theta^{*}\right)=\right.$ $0.25]$, rain water begins to pool on the soil surface rather than infiltrating the soils. As time goes on, the ponding water adds more hydraulic pressure on the soil surface, which finally overcomes the soil water repellency and drives the formation of water channels. Only one or two water channels are seen in these cases, even in a 55-cm-wide sample cell.

Figure 6 summarizes the results obtained from both hydrophilic samples (solid squares) and partially hydrophobic ones (open stars) at a fixed rain condition. The expression in the $x$ axis has a physical meaning of capillary rise height, which combines the influence of bead diameter $D$ and the effective contact angle $\theta^{*}$. It is the natural length scale to consider. In Fig. 6(a), we see that the infiltration depth in a soil sample grows almost linearly as the capillary rise height of that sample increases. A linear fit on the data gives us a slope of $\alpha=0.34$. Figure 6(b) shows the variation of the water channel width. As the capillary rise height of a soil increases, the water channel width increases quicker than the infiltration depth. A power-law fit is applied to the data and obtains a power value of $\delta=2.26$. Figure 6(c) shows the variation of channel separation. For hydrophilic samples, it shows a gentle decrease with decreasing bead diameter, but for increasing soil hydrophobicity, it shows a rapid decrease with the capillary rise length.

Among the parameters we discuss above, the water channel width is the only one that has been widely studied in the literature $[7,14,16,17,24,25,27]$. Chuoke et al. [24] 


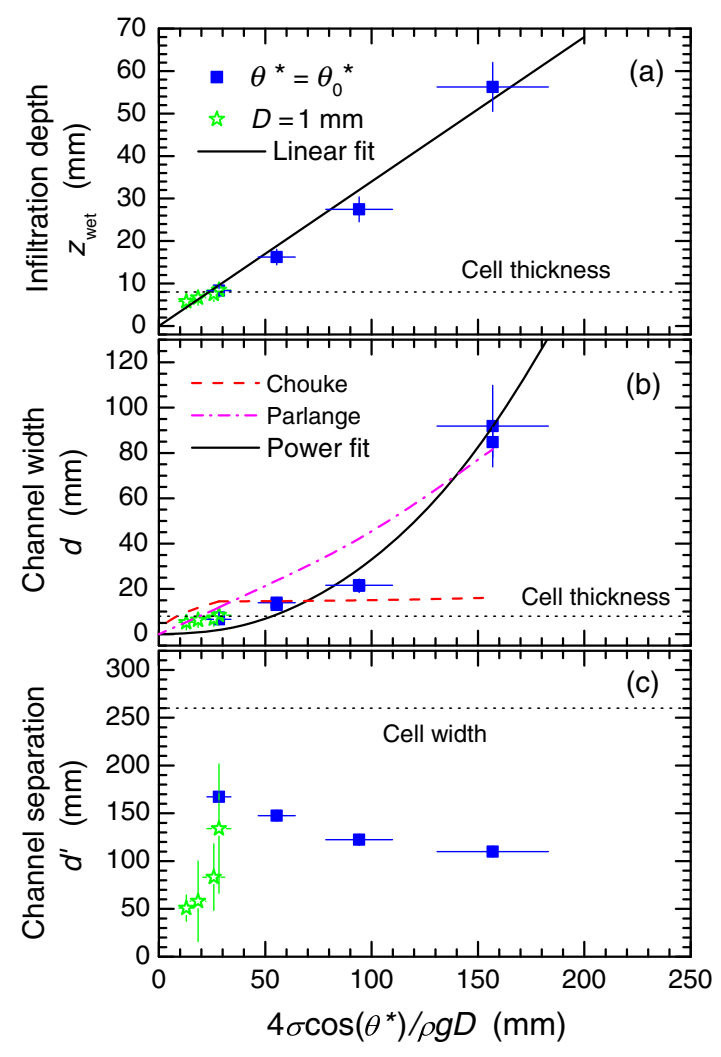

FIG. 6. Variation of (a) infiltration depth $z_{\text {wet }}$, (b) channel width $d$, and (c) channel separation $d^{\prime}$, with the capillary rise height of the randomly close-packed glass beads $\left[4 \sigma \cos \left(\theta^{*}\right) / \rho g D\right]$ at a fixed rain condition. The parameters plotted here are defined in Fig. 2. The rain rate is $Q=14.5 \mathrm{~cm} / \mathrm{h}$, and the raindrop impinging speed is $U_{T}=1.0 \mathrm{~m} / \mathrm{s}$. In plots (a)-(c), solid squares represent the data obtained from hydrophilic glass beads with varied diameters (fix $\theta^{*}$ and vary $D$ ), while open stars represent data obtained from 1-mm glass beads with varied surface wetting properties (fix $D$ and vary $\theta^{*}$ ). Solid curve in (a) is a linearly fit to the data. Solid curve in (b) is a power-law fit, and the dashed curves are predictions from Eqs. (4) and (7), respectively.

assumed a Laplace-Young relationship for the pressure jump across the horizonal wetting front and used a stability analysis to obtain an expression of the channel width as

$$
d=\pi\left(\frac{3 \sigma \cos \left(\theta^{*}\right)}{\rho g} \frac{1}{1-Q / \kappa_{f}}\right)^{1 / 2} .
$$

Here, $\sigma$ is the surface tension of the applied rain water, and $\kappa_{f}$ is the measured hydraulic conductivity inside the water channel, whose value is usually a bit less or equal to the saturated hydraulic conductivity of the soil. We assume $\kappa_{f} \approx \kappa_{s}$ and add the prediction of Eq. (4) to Fig. 6(b) in the dashed line. We see that Chuoke's model significantly deviates from our experimental data, which is consistent with the conclusions Glass et al. drew in their infiltration experiments on sands [27].
Parlange and Hill [25] later derived a relationship between wetting front velocity and curvature and applied that in a stability analysis to obtain an expression of

$$
d=\pi \frac{s_{w}^{2}}{\kappa_{s}\left(S_{s}-S_{0}\right)} \frac{1}{\left(1-Q / \kappa_{f}\right)} .
$$

Here, $S$ is the so-called water content and defined as the ratio of water volume retained in a soil and the total volume of the soil. The subscripts $s$ and 0 represent the saturated state and the initial state of the soil, respectively. For data shown in Fig. 6(b), we have $S_{0}=0$ (dry in initial state) and $S_{s}=\epsilon \approx 0.36$ ( $\epsilon$ is the porosity of the sandy soil).

In Eq. (5), $s_{w}$ is the soil water sorptivity, which has a unit of length over the square root of time and measures the capacity of soils to absorb water through capillarity. Culligan et al. [31] applied scaling analysis and showed that the soil water sorptivity can be calculated as

$$
s_{w}=s^{*}\left(\frac{\epsilon l^{*}}{\mu} \sigma \cos \left(\theta^{*}\right)\right)^{1 / 2} .
$$

Here, $s^{*}$ is a dimensionless parameter called intrinsic sorptivity, and its value is determined as $s^{*}=0.133$ by Parlange and co-worker [32]. $l^{*}$ is the characteristic pore radius in sandy soils, and it is proportional to the diameter $D$ of soil grains. We assume $l^{*}=\beta(D)$ and $\kappa_{f} \approx \kappa_{s}$. Take Eq. (6) into Eq. (5) and we have

$$
d=\beta \frac{\pi\left(s^{*}\right)^{2} \sigma \cos \left(\theta^{*}\right)}{\rho g D K_{0}} \frac{1}{\left(1-Q / \kappa_{s}\right)} .
$$

We fit the data in Fig. 6(b) using Eq. (7) and determine that $\beta=0.019$. One may notice that the characteristic pore radius obtained here is far smaller that that determined by the pressure-plate method [4]. The reason is when writing down Eq. (6), we ignore a term that reflects the saturation level in water channels and combine its influence into the fitting parameter $\beta$. Comparing the predictions from different models and a simple power-law fit, we find that Parlange's model describes the experimental data better than Chuoke's model, which is consistent with the previous literature $[7,14,16,27]$; however, the power-law fit offers an excellent empirical description.

\section{ENHANCING THE RAIN-WATER-REACHABLE AREA IN SANDY SOILS}

\section{A. Prewetting sandy soils}

To improve the usage of rain and irrigation water by plants, an important strategy is to suppress or to modify rain water channeling behavior. To suppress the water channeling behavior, a possible method is to prewet dry sandy soil a bit before rain. Lu et al. [33] observed that water channels initiated in a dry zone vanish in a prewet zone for a $2 \mathrm{D}$ 
layered glass bead packing. Later, Bauters et al. [34] determined that the width of the water channel formed in prewet quartz sand under a point water source shows strong dependence on the initial water content. Here, we systematically extract the steady-state wetting patterns of a prewet model sandy soil under nonponding rainfall using 1-mm hydrophilic glass beads. For this, different amounts of water are uniformly mixed into air-dried glass beads and then the partially wet beads are closely packed into a sample cell. The initial water content $S_{0}$ in each packing is determined as the ratio of the added water volume and the total volume of the glass bead packing. To estimate the socalled field capacity of the model sandy soil, which is the maximum amount of water a soil can hold in freely draining condition, we fill the sample cell with a fixed amount of air-dried model sandy soil and then slowly immerse it into water to fully saturate the soil sample. After that, the sample cell is moved out carefully and suspended in air for an hour of free draining. The wet soil sample is then poured out, and the mass of the soil sample is measured. By comparing the masses for wet versus dry, we determine that the field capacity of the model sandy soil is around $7.75 \%$, which is only about one-fifth of its full saturation value of $36 \%$. The value of the field capacity set the upper limit of the initial water content we apply.

In the experiments, we set the rain rate to $Q=$ $14.5 \mathrm{~cm} / \mathrm{h}$ and the raindrop impinging speed to $U_{T}=1 \mathrm{~m} / \mathrm{s}$. The steady-state wetting pattern at each initial water-content value is obtained by subtracting the background image captured at the rain start from that captured at the steady state and then by converting to gray scale with enhanced contrast. Figures $7(a)-7(d)$ show the steady-state wetting patterns for a model sandy soil with initial water content $S_{0}$ equal to $0,2.5 \%, 5.0 \%$, and $7.5 \%$, respectively. We see more water channels forming when the initial water content $S_{0}$ increases. Differing from the simulation results from Juanes and co-worker [28], the uniform infiltration depth does not show a significant increase when the initial water content in the soil increases. Water channels formed in prewet soil have very irregular shapes and sometimes they even branch or entangle. The equivalent channel width looks larger for soil with a higher initial water content, which is consistent with the observation of Bauters et al. [34]. For soil with an initial water content close to its field capacity, rain water is able to reach almost everywhere in the soil packing. Figure 7(e) shows the steady-state wetting pattern of rain water in a model soil packing with an initial water content equal to its field capacity. This packing is prepared in the same way as we determined the soil field capacity. It is used to exclude the possible structure heterogeneity due to the packing method. We see that it is very similar to Fig. 7(d). Instead of forming water channels, the rain water flows throughout the soil packing. Since prewet soil can effectively suppress rain water channeling and enlarge the rain-water-reachable region in sandy soils, a good way to increase the usage of irrigation water by plants is to monitor the variation of the water content in soils with time and manage the irrigation time period based on that. To prewet the field may not sound practical, but real soils in the field are never perfectly dry. They usually have a nonzero water content that drops as time passes. Our study points to a way to determine the critical value of water content in soils that can significantly suppress water channel formation. To achieve irrigation efficiency, the field should be irrigated at the time when its soil water content drops below the critical value.

\section{B. Modifying surface flatness of sandy soils}

Another method is to create an extra hydraulic pressure gradient on the horizontal wetting front to control the location and the separation of water channels. By modifying the surface flatness of the soil packing, we are able to alter the shape of the wetting front away from horizontal. Because of gravity, the hydraulic pressure on the bottom of the hill is higher than that at other locations. Thus, water channels should prefer to form there. To our knowledge, this method has not been tested or reported before. Figure 8 shows the steady-state wetting pattern in a dry model sandy soil, hydrophilic 1-mm glass beads, at four different test runs. In each test, the rain conditions are the same. The soil surface shapes are the same in test 2 and test 3; the soil surface shapes for other tests are different. We indeed find a curved wetting front in every test, such that the shape of the wetting front follows the shape of the soil surface. In test (a) $S_{0}=0$

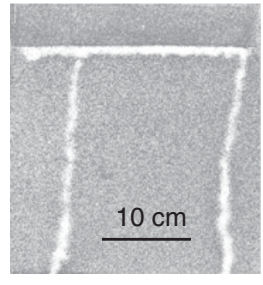

(b) $S_{0}=2.5 \%$

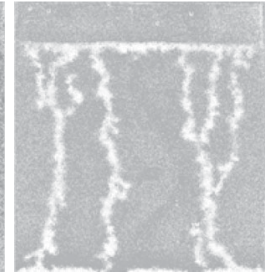

(c) $S_{0}=5.0 \%$

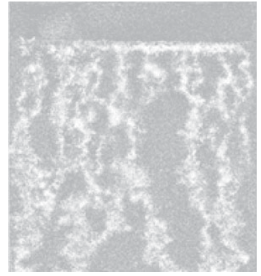

(d) $S_{0}=7.5 \%$

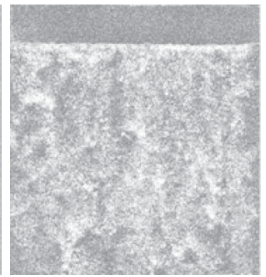

(e) $S_{0}=7.55 \%$

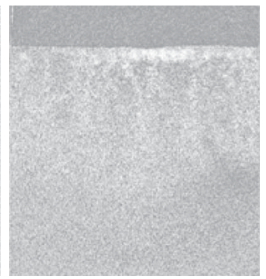

FIG. 7. Gray-scale images (a)-(e) showing the influence of initial water content $S_{0}$ increasing from 0 to filled capacity, as labeled, on rain water channeling in a model sandy soil, 1-mm hydrophilic glass beads, at a fixed rain condition. The rain rate is $Q=14.5 \mathrm{~cm} / \mathrm{h}$, and the raindrop impinging speed is $U_{T}=1 \mathrm{~m} / \mathrm{s}$. The sample packing is $26 \mathrm{~cm}$ wide and $0.8 \mathrm{~cm}$ thick. The sample packing height is around $25 \mathrm{~cm}$. In this model sandy soil, the saturated water content is around $36 \%$, and the field capacity is around $7.75 \%$. 


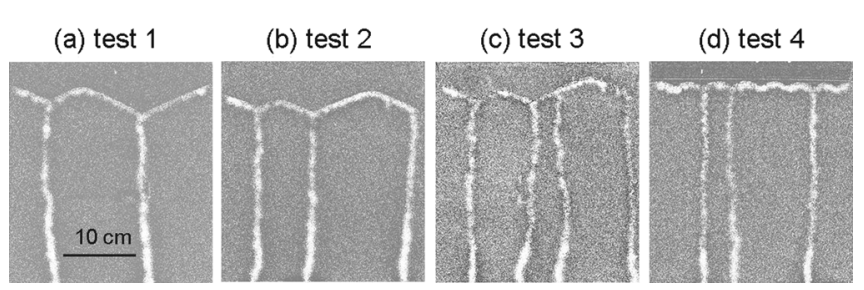

FIG. 8. Gray-scale images (a)-(d) showing the influence of four different surface flatnesses, as labeled, on rain water channeling in a dry model sandy soil, 1-mm hydrophilic glass beads at a fixed rain condition. The rain rate is $Q=14.5 \mathrm{~cm} / \mathrm{h}$, and the raindrop impinging speed is $U_{T}=1 \mathrm{~m} / \mathrm{s}$. The sample packing is $26 \mathrm{~cm}$ wide and $0.8 \mathrm{~cm}$ thick. The sample packing height varies due to the wavelike surface. More water channels may form when the wavelike surface creates an extra hydraulic pressure gradient on the wetting front.

1 and test 2, water channels form at the bottoms of the hills, as expected, but in test 3 , we see an extra channel forming on the slope of the hill, and in test 4, the soil surface is curved like a small wave with seven bottoms, but only three channels finally grow. These experiments show that we can increase the number of water channels in dry sandy soils by creating large enough curvatures on the soil surface, but we cannot precisely control their locations and numbers.

\section{Adding superabsorbent hydrogel particles}

As discussed above, by prewetting the soil or curving the soil surface, we can enlarge the rain-water-reachable region in sandy soils. However, due to the high hydraulic conductivity in sandy soils, that amount of water will flow out quickly from the plant root zone unless soil additives are applied to help hold it in place. Superabsorbent hydrogel particle additives are introduced for this purpose, and previous studies have proven their efficiency in reducing hydraulic conductivity in sandy soil and enhancing sandy soil water retention [1,2,4,35-39]. The hydrogel particle additives we use here are provided by Degussa Inc. (Stockosorb SW). The main chemical component of the product is cross-linked potassium acrylate and acrylamide at a ratio of 50/50. The dry hydrogel particles have a faceted shape and are sieved between $0.3-$ and $0.5-\mathrm{mm}$ sized meshes. When freely bathed with water, they swell to about 5 times their original diameter within about $3 \mathrm{~h}$. If the swollen hydrogel particles are then exposed in atmosphere at room temperature, we find that they deswell slowly and lose over $80 \%$ of their stored water within a day. These hydrogel particles are able to repeat the swelling-todeswelling cycle many times without decomposition.

Our previous 3D rain experiments [18] showed that these hydrogel particle additives significantly affect the transport and storage of rain water in sandy soils, and their effects strongly depend on their distribution methods. Here, using the same 2D setup as above, we are now able to visualize the time evolution of hydrogel particle additives in sandy soil under rainfall. Two different methods are applied to distribute dry hydrogel particles into a dry model sandy soil, 1-mm hydrophilic glass beads: one is uniformly mixing and the other is placing them in a layer underground. Experiments are performed at a rain rate of $Q=$ $14.5 \mathrm{~cm} / \mathrm{h}$ and a raindrop impinging speed of $U_{T}=1 \mathrm{~m} / \mathrm{s}$ for over $5 \mathrm{~h}$. Dry hydrogel particles are white and too small to be seen when mixed into the soil, but after swelling, they become large and transparent. The back light passes through them to create bright spots, which can be easily identified in the raw images.

Figures 9(a)-9(e) show an image sequence captured from a 1-mm hydrophilic glass bead packing with $0.1 \mathrm{wt} \%$ dry hydrogel particles uniformly mixed into the top $10-\mathrm{cm}$ region. A red line is glued on the outside surface of the sample cell to mark the boundary of the mixture and the pure model soil. We see that in this packing, water channels form and grow the same as in a "no gel" packing at the early time of rain. A bit later, hydrogel particles that are located at the top wet layer or in the water channels swell to a detectable size. They begin to perturb the soil surface and extend the water channel above the red line. As time goes on, the size of the swollen hydrogel particles keeps growing, and the width of the water channels keeps enlarging. Dry hydrogel particles near the water channel (a) $t=1 \min$

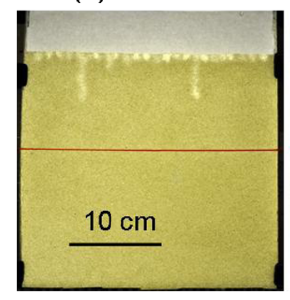

(b) $t=2 \min$

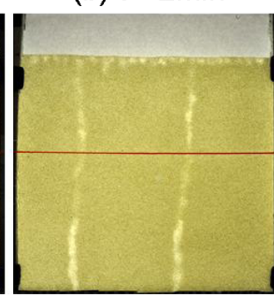

(c) $\mathrm{t}=30 \mathrm{~min}$

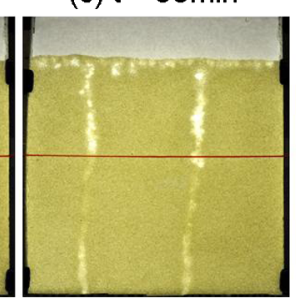

(d) $t=60 \mathrm{~min}$

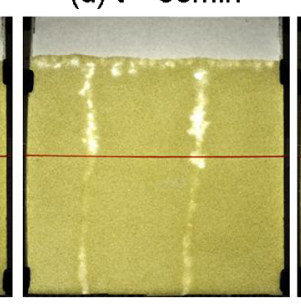

(e) $t=300 \mathrm{~min}$

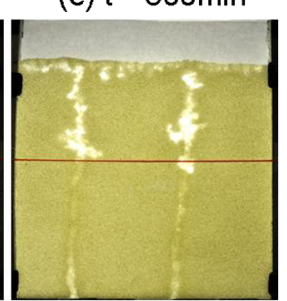

FIG. 9. Sequence of gray-scale images (a)-(e) at different times, as labeled, showing the influence of uniformly mixed hydrogel particle additives on the rain water channeling in a sandy soil composed of 1-mm hydrophilic glass beads; $0.1 \mathrm{wt} \%$ of dry hydrogel particles $(0.3-0.5 \mathrm{~mm}$ in axis) are uniformly mixed into the top $10 \mathrm{~cm}$ of the dry model sandy soil before the rain. The rain rate is $Q=14.5 \mathrm{~cm} / \mathrm{h}$, and the raindrop impinging speed is $U_{T}=1 \mathrm{~m} / \mathrm{s}$. The sample packing is $26 \mathrm{~cm}$ wide and $0.8 \mathrm{~cm}$ thick. The sample packing height is $25 \mathrm{~cm}$ (dry). The red line in each image indicates the boundary of the mixture and the pure model soil. The swelling of hydrogel particles extends the water channels. 


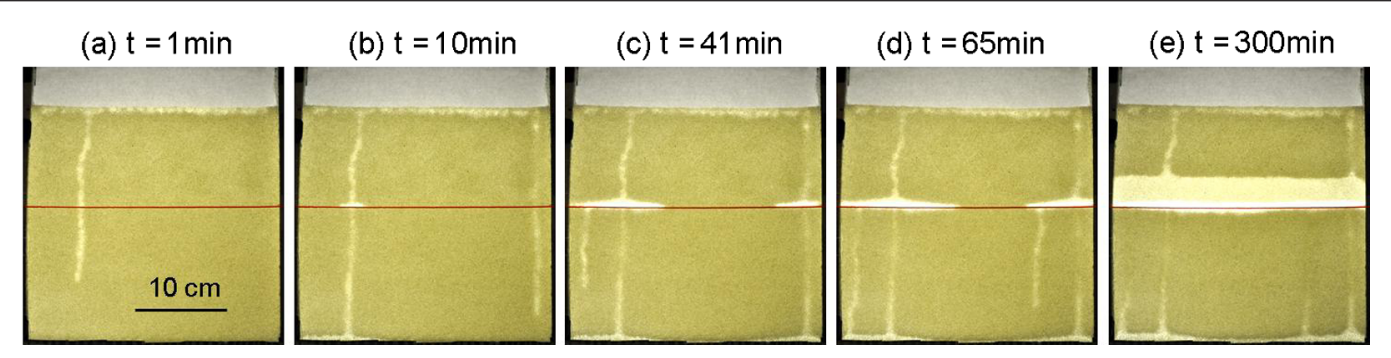

FIG. 10. Sequence of gray-scale images (a)-(e) at different times, as labeled, showing the influence of a layer of hydrogel particle additives on the rain water channeling in a sandy soil composed of 1-mm hydrophilic glass beads; $0.1 \mathrm{~g}$ of dry hydrogel particles $(0.3-0.5 \mathrm{~mm}$ in axis) are placed in a layer under the top $10 \mathrm{~cm}$ of the dry model sandy soil before the rain. The rain rate is $Q=14.5 \mathrm{~cm} / \mathrm{h}$, and the raindrop impinging speed is $U_{T}=1 \mathrm{~m} / \mathrm{s}$. The sample packing is $26 \mathrm{~cm}$ wide and $0.8 \mathrm{~cm}$ thick. The sample packing height is $25 \mathrm{~cm}$ (dry). The red line in each image indicates the location of the hydrogel particles. The swollen hydrogel particles partially clog the water channels, form a wet hydrogel layer across the sample packing, and create a fully saturated region in the soil above them.

now contact rain water. Finally, the water channels show very irregular shapes in the mixture region but keep the initial shape on the pure soil region. Several conclusions are drawn from this experiment. First, when mixed into soils, hydrogel particles enhance the soil water retention by extending the well-established water channels rather than by modifying the water channel formation process. The reason is that the swelling time scale of the hydrogel particles is much slower than that of the water channel formation. Second, within the range of concentration used here, the swollen hydrogel particles appearing in water channels cannot effectively clog them and reduce their hydraulic conductivity. Third, rain water channeling keeps most of the hydrogel particle additives away from rain water; thus, uniformly mixing a small percentage of dry hydrogel particles into sandy soils may significantly lower the efficiency of this product on improving soil water retention.

Figures 10(a)-10(e) are image sequences captured from a 1-mm hydrophilic glass bead packing with $0.1 \mathrm{~g}$ of dry hydrogel particles placed in a layer under the top $10 \mathrm{~cm}$ of glass beads. The red line shown in each image is glued on the outside of the sample cell, and it marks the location of the hydrogel particles in the model sandy soil packing. We see that a water channel grows first after about $1 \mathrm{~min}$ of rain, and a second one grows about 10 min later. This situation happens commonly in pure model sandy soils. The only difference is that a wet gel layer grows horizontally along the red line where the dry hydrogel particles are placed. As time goes on, the building wet gel layer begins to partially clog the water channels; thus, two extra water channels form on the tips of the wet gel layer to drain out rain water. When a continuous wet gel layer has formed, rain water begins to accumulate above it, and a fully saturated region grows upward in the model sandy soil. Finally, the thickness of the wet gel layer and the depth of the saturated-soil region stop growing and a steady state is achieved. In the steady state, the wet gel layer lifts the model sandy soil up a bit, all the added hydrogel particles are in swollen states, and some extra rain water is stored in the model sandy soil pores. After the rain stops, the rain water stored in the model sandy soil keeps draining out slowly, but the water held by the hydrogel particles stays for a long time. This experiment clearly demonstrates that placing hydrogel particles in a layer underground is a more efficient way to use this type of soil additive, especially when water channeling occurs in soils during rain or irrigation.

\section{CONCLUSION}

In summary, we investigate the phenomenon of rain water channeling in systematically varied model sandy soils that have well-established pore structure and surface wetting properties at different rain conditions. In a homogenous sandy soil packing, the formation of water channels is caused by the instabilities occurring at the horizontal wetting front. By visualizing water channels in sandy soils with different grain diameter and surface wetting properties at different rain conditions, we determine the relationships between the infiltration depth, the water channel width, the channel separation with the rain conditions, and the capillary forces in model sandy soils. The raindrop impinging speed has little influence on water channel formation, but the rain rate strongly affects water channel size and separation. The infiltration depth increases linearly as capillary forces increase; the water channel width increases even quicker than the infiltration depth and is well described by a power law; the channel separation shows almost no dependence on the grain diameter but is very sensitive to even a small change on the soil wetting property. Among these parameters, the water channel width is the only one that has been frequently discussed in the literature. We compare our results to the former predictions on the water channel width and find that our results obey the most commonly used predictions from Parlange and Hill [25].

With this understanding on rain water channeling in sandy soils, we then study the efficiency of different methods on improving rain water retention. By prewetting 
a dry model sandy soil at different levels before rain, we can increase the number of water channels and enlarge the channel width and, thus, extend the rain-water-reachable region. By curving the soil surface to wavelike shapes, we can control the shape of the wetting front formed under rainfall and, thus, create more water channels in dry sandy soils. By adding superabsorbent hydrogel particles, we can modify the water channels in sandy soils in different ways by using different distribution methods. Since the swelling time of hydrogel particles is far longer than that of the water channel formation, mixing a small amount into sandy soils has almost no effect on the formation of water channels in the early time of rain. However, as time passes, the swelling of hydrogel particles perturbs the soil structure and changes the wetting pattern in soils. When uniformly mixed in soil, only the hydrogel particles that reside in the top wet layer or within the water channels are able to contact rain water. Their swelling extends the water channels. When placed in a layer underground, by contrast, all the hydrogel particles may be able to contact rain water sooner or later. They not only swell to hold rain water inside but also partially clog water channels and cause accumulation of rain water in the soil above. Comparing the two methods, the latter one is a more efficient way to use hydrogel particle additives.

\section{ACKNOWLEDGMENTS}

We thank Jean-Christophe Castaing, Larry Hough, and Zhiyun Chen from Solvay-Rhodia Inc. for helpful discussions. We also thank Christian Fretigny for suggestions. This work is supported by the National Science Foundation through Grants No. MRSEC/DMR-1120901 (Y. W. and D. J. D.) and No. DMR-1305199 (D. J. D.).

[1] A. K. Bhardwaj, I. Shainberg, D. Goldstein, D. N. Warrington, and G. J. Levy, Water retention and hydraulic conductivity of cross-linked polyacrylamides in sandy soils, Soil Sci. Soc. Am. J. 71, 406 (2007).

[2] W. Bai, H. Zhang, B. Liu, Y. Wu, and J. Song, Effects of super-absorbent polymers on the physical and chemical properties of soil following different wetting and drying cycles, Soil Use Manage. 26, 253 (2010).

[3] E. Verneuil and D. J. Durian, Permeability of mixed soft and hard granular material: Hydrogels as drainage modifiers, Eur. Phys. J. E 34, 65 (2011).

[4] Y. Wei and D. J. Durian, Effect of hydrogel particle additives on water-accessible pore structure of sandy soils: A custom pressure plate apparatus and capillary bundle model, Phys. Rev. E 87, 053013 (2013).

[5] D. E. Hill and J. Y. Parlange, Wetting front instability in layered soils, Soil Sci. Soc. Am. J. 36, 697 (1972).

[6] G. A. Diment and K. K. Watson, Stability analysis of watermovement in unsaturated porous materials.3. experimental studies, Water Resour. Res. 21, 979 (1985).

[7] R. J. Glass, T. S. Steenhuis, and J. Y. Parlange, Wetting front instability.2. experimental-determination of relationships between system parameters and two-dimensional unstable flow field behavior in initially dry porous-media, Water Resour. Res. 25, 1195 (1989).

[8] R. S. Baker and D. Hillel, Laboratory tests of a theory of fingering during infiltration into layered soils, Soil Sci. Soc. Am. J. 54, 20 (1990).

[9] H. C. Vanommen, R. Dijksma, J. M. H. Hendrickx, L. W. Dekker, J. Hulshof, and M. Vandenheuvel, Experimental assessment of preferential flow paths in a field soil, J. Hydrol. 105, 253 (1989).

[10] C. J. Ritsema, L. W. Dekker, J. M. H. Hendrickx, and W. Hamminga, Preferential flow mechanism in a water repellent sandy soil, Water Resour. Res. 29, 2183 (1993).

[11] J. M. H. Hendrickx, L. W. Dekker, and O. H. Boersma, Unstable wetting fronts in water-repellent field soils, J. Environ. Qual. 22, 109 (1993).

[12] C. J. Ritsema, L. W. Dekker, J. L. Nieber, and T. S. Steenhuis, Modeling and field evidence of finger formation and finger recurrence in a water repellent sandy soil, Water Resour. Res. 34, 555 (1998).

[13] A. Williams, D. Scholefield, J. Dowd, N. Holden, and L. Deeks, Investigating preferential flow in a large intact soil block under pasture, Soil Use Manage. 16, 264 (2000).

[14] J. S. Selker, T. S. Steenhuis, and J. Y. Parlange, Wetting front instability in homogeneous sandy soils under continuous infiltration, Soil Sci. Soc. Am. J. 56, 1346 (1992).

[15] M. S. Babel, R. Loof, and A. Dasgupta, Fingered preferential flow in unsaturated homogeneous coarse sands, Hydrol. Sci. J. 40, 1 (1995).

[16] T. M. Yao and J.M. H. Hendrickx, Stability of wetting fronts in dry homogeneous soils under low infiltration rates, Soil Sci. Soc. Am. J. 60, 20 (1996).

[17] T. Annaka and S. Hanayama, Effects of contact angle on fingered flow during non-ponding infiltration into dry sand layers, Soil Sci. Plant Nutr. 56, 366 (2010).

[18] Y. Wei and D. J. Durian, arXiv:1310.6258.

[19] C. M. Cejas, Y. Wei, R. Barrois, C. Fretigny, D. J. Durian, and R. Dreyfus, arXiv:1403.3364.

[20] G. S. Beavers, E. M. Sparrow, and D. E. Rodenz, Influence of bed size on flow characteristics and porosity of randomly packed-beds of spheres, J. Appl. Mech. 40, 655 (1973).

[21] H. Katsuragi, Morphology scaling of drop impact onto a granular layer, Phys. Rev. Lett. 104, 218001 (2010).

[22] G. Delon, D. Terwagne, S. Dorbolo, N. Vandewalle, and H. Caps, Impact of liquid droplets on granular media, Phys. Rev. E 84, 046320 (2011).

[23] N. Cheng, Formula for the viscosity of a glycerol-water mixture, Ind. Eng. Chem. Res. 47, 3285 (2008).

[24] R. L. Chuoke, P. van Meurs, and C. van der Poel, The instability of slow, immiscible, viscous liquid-liquid displacements in permeable media, Petrol. Trans. Am. Inst. Min. Metall. Eng. 216, 188 (1959).

[25] J. Y. Parlange and D. E. Hill, Theoretical-analysis of wetting front instability in soils, Soil Science 122, 236 (1976).

[26] R. J. Glass, J. Y. Parlange, and T. S. Steenhuis, Wetting front instability.1. theoretical discussion and dimensional analysis, Water Resour. Res. 25, 1187 (1989).

[27] R. J. Glass, J. Y. Parlange, and T. S. Steenhuis, Immiscible displacement in porous-media-stability analysis of 3dimensional, axisymmetrical disturbances with application 
to gravity-driven wetting front instability, Water Resour. Res. 27, 1947 (1991).

[28] L. Cueto-Felgueroso and R. Juanes, Nonlocal interface dynamics and pattern formation in gravity-driven unsaturated flow through porous media, Phys. Rev. Lett. 101, 244504 (2008).

[29] D. Shchekotov, Master's thesis, University of Stavanger, 2010.

[30] D. J. Durian and C. Franck, Wetting phenomena of binaryliquid mixtures on chemically altered substrates, Phys. Rev. Lett. 59, 555 (1987).

[31] P. J. Culligan, V. Ivanov, and J. T. Germaine, Sorptivity and liquid infiltration into dry soil, Adv. Water Resour. 28, 1010 (2005).

[32] D. A. Lockington and J. Y. Parlange, Anomalous water absorption in porous materials, J. Phys. D 36, 760 (2003).

[33] T. X. Lu, J. W. Biggar, and D. R. Nielsen, Water-movement in glass bead porous-media.2. experiments of infiltration and finger flow, Water Resour. Res. 30, 3283 (1994).
[34] T. W. J. Bauters, D. A. DiCarlo, T. S. Steenhuis, and J. Y. Parlange, Soil water content dependent wetting front characteristics in sands, J. Hydrol. 231-232, 244 (2000).

[35] R. A. I. Azzam, Polymeric conditioner gels for desert soils, Commun. Soil Sci. Plant Anal. 14, 739 (1983).

[36] R. L. Flannery and W. J. Busscher, Use of a syntheticpolymer in potting soils to improve water holding capacity, Commun. Soil Sci. Plant Anal. 13, 103 (1982).

[37] M. S. Johnson, The effects of gel-forming polyacrylamides on moisture storage in sandy soils, J. Sci. Food Agric. 35, 1196 (1984).

[38] D. L. Bouranis, A. G. Theodoropoulos, and J. B. Drossopoulos, Designing synthetic-polymers as soil conditioners, Commun. Soil Sci. Plant Anal. 26, 1455 (1995).

[39] J. Abedi-Koupai, F. Sohrab, and G. Swarbrick, Evaluation of hydrogel application on soil water retention characteristics, J. Plant Nutr. 31, 317 (2008). 